\title{
Die Alquié-Alexander'sche Operation zur Beseitigung des Prolapsus und der Retroflexio nteri nach Kocher ${ }^{1}$ ).
}

\author{
Von \\ Dr. Otto Lanz, \\ Erster Assistent an der chirurgischen Klinik in Bern.
}

(Mit 2 Abbildungen im 'lext.)

Durch zahlreiche Publikationen über die operative Behandlung der Retrodeviationen aus den letzten Jahren, sowie durch die Discussionen über dasselbe Thema an den Gynäkologen-Congressen in München und Freiburg, auf der Naturforscher-Versammlung in Heidelberg, in der gynäkologischen Gesellschaft zu Berlin etc. hat diese Frage erhöhtes Interesse gewonnen. Dasselbe ist nicht unerklärlich, wenn wir die Wichtigkeit und Ausdehnung des Leidens vergleichen mit den mangelhaften Heilungsresultaten. Sind ja doch nach Sänger 1/6 aller gynäkologischen Fälle Retroflexionen und Löhlein kommt zu dem Schlusse, dass die Heilung derselben durch die actuellen therapeutischen Maassnahmen die Ausnahme sei.

Das Verdienst, die normale Lage des Uterus festgestellt zu haben, gebührt B. Schultze, indem er angab, die Normalstellung des Uterus sei die, dass die Cervix nach dem Sacrum, der Fundus uteri nach der Symphyse zu gerichtet sei. Erst auf Grund dieser neuen Anschauung wurde dann der Symptomencomplex der Retrodeviation: die chronischen Reizzustände des Uterus, die Neigung zu Endometritiden und anderen entzündlichen Affectionen, dysmenorrhoische Beschwerden, Menorrhagieen, Sterilität, mechanische

1) Die Arbeit wurde abgeschlossen am 30. 9. 1892. 
Belästigung der Nachbarorgane, chron. Obstipation, Gefühl der Schwere im Becken, Kreuzschmerzen, Lähmungserscheinungen und Neurosen als auf einheitlicher Basis beruhend, anerkannt.

Trotzdem jedoch diese Lehre von den Lageveränderungen des Uterus relativ jungen Datums ist, sind die Behandlungsmethoden ausserordentlich zahlreich und es werden deren immer wieder neue angegeben, was uns einerseits die sehr grosse Bedeutung des bekämpften Leidens zeigt, andrerseits die Wahrheit der von Roux aufgestellten Behauptung beweist: pour le traitement des déviations utérines la richesse de notre arsenal thérapeutique démontre notre impuissance.

Anfangs war die Behandlung der Retrodeviationen eine ausschliesslich orthopädische und es wurden gegen dieselben der Reihe nach die ganze Serie der Pessarien ins Feld geführt. Und in der That liegt ja die orthopädische Behandlung am nächsten, sobald die erste Frage bei der Inangriffnahme jeder Therapie, diejenige, ob der Uterus reponirbar ist, im bejahenden Sinne erledigt ist. Ist dies der Fall, so wird er manuell oder mit der Sonde reponirt und jetzt wird es sich allerdings um den Versuch handeln, den Uterus in normaler Lage durch ein Pessar zu fixiren. Allein abgesehen davon, dass eine orthopädische Behandlung niemals das Ideal der Therapie ist und dass mir wenigstens Terrier's „, horreur instinctive" vor den Pessarien in diesem speciellen Falle nicht ganz unbegreiflich erscheint, kann die Pessarbehandlung durchaus nicht den Werth einer specifischen Behandlungsmethode beanspruchen, wenn auch Schröder die Prognose der bleibenden Heilung bei Pessarbehándlung, wie Mundé und Löhlein sie stellen, für zu ungünstig hält und angiebt: „ich selbst sehe es doch nicht ganz selten, dass nach jáhrelangem Tragen eines geeigneten Pessars der Uterus auch nach Entfernung desselben dauernd liegen bleibt." Wenn man aber bedenkt, dass ein Mann wie Schröder sich auf solche Weise ausdrückt, welche Resultate stehen dann von den Pessarien in der Hand Ungeübter zu erwarten. Erklärt doch sogar Fritsch auf dem zweiten Gynäkologen-Congress in Halle 1888, dass er 10 Jahre gebraucht habe, um ein Pessar anlegen zu lernen, und dass er noch jotzt eine Woche brauche, um das geeignete ausfindig zu machen.

Nach Untersuchungen von Küstner stammt ein viel grösserer Bruchtheil der Retroflexionen aus dem kindlichen Alter und der Zeit der Pubertät her, als man bisher annahm und da Veit nach- 
gewiesen hat, dass die Heilbarkeit der Retroflexio bei ganz jugendlichen Frauen die besten Aussichten hat, während sich dieselben von den dreissiger Jahren an, zumal gegen das Klimakterium hin verschlechtern, so wird man wohl nach wie vor bei jüngeren Individuen die Pessarbehandlung mit einer gewissen Aussicht auf Radicalheilung versuchen können, falls dieselbe nicht durch ein idealeres Verfahren verdrängt wird, das auf Grund seiner Vorzüge zu allgemeiner Anerkennung gelangt.

Die orthopädische Behandlung der Lageveränderungen ist, wie schon angedeutet, nur dann am Platze, wenn der Uterus frei beweglich ist; andernfalls muss er zuerst durch Massage und resorptionsbefördernde Kuren beweglich gemacht werden: die ThureBrandt'sche Methode, die forcirte Reposition nach Schultze verfolgen diesen Zweck ohne operativen Eingriff; in analoger Weise geht Vuillet vor, indem er den Uterus nach seiner Methode so weit dilatirt, dass er den Finger einführen kann und dann unter Mithülfe der anderen Hand bei Adhäsionen des retroflectirten Uterus löst, während Sims die Hysterotomia interna vorschlägt und Köberle die Laparotomie macht und nun die pelveoperitonitischen Stränge, die den Uterus fixiren, löst.

Die Frage nach der operativen Behandlung der Retroflexionen des Uterus erhielt erst durch Alexander eine neue Anregung und erst seither und durch die später aufkommende Ventrofixation haben die Lageveränderungen auch chirurgisches Interesse gewonnen.

Der Gedanke, durch Zug der Ligamenta rotunda den prolabirten Uterus zu reponiren, gehört Alquié 1840, während Aran zuerst den Gedanken äusserte, bei Retroflexion dadurch den Fundus uteri aufzurichten. Alexander und nach ihm Adams gebührt das Verdienst, die Verkürzung der Ligamenta rotunda als Operationsmethode eingeführt und eingebürgert zu haben; denn dass dem Gedanken von Alquié und Aran sogar in ihrem Mutterlande wenig Wichtigkeit beigemessen wurde, ersehen wir aus Tillaux, der in seiner topographischen Anatomie noch im Jahre 1884 schreibt: M. Alquié pensant que les ligaments étaient la cause de la résistence que l'on éprouve parfois à abaisser la matrice, avait eu la singulière idée de les raccourrir pour s'opposer anx chutes de cet organe; d'autre part, Aran, attribuant à ces mêmes ligaments le rôle non de suspendre l'utérus, mais d'en attirer le fond en avant, avait songé à appliquer l'opération de M. Alquié à la 
cure de la rétroflexion. Je ne sache pas que la pratique soit venue confirmer ces idées purement théoriques."

Unter anderen Namen, die mit dieser Operation in Zusammenhang stehen, finden wir B. von Langenbeck 1856 (nach einer mündlichen Mittheilung von Dr. Reynier an Prof. Roux), Deneffe 1864, Rivington und Freund.

Da Alquié die Priorität der Idee, Alexander das Hauptverdienst ihrer Einführung in die operative Praxis zukommt, wäre es wohl am passendsten, die Methode als Alquié-Alexander'sche Operation zu bezeichnen.

Die „singulière idée" Alquié's hat, auch nachdem sie durch Alexander und Adams exact ausgebildet and in ihrer Wirkung studirt worden ist, unter den Gynäkologen Deutschlands wenig Anklang gefunden. Bevor wir auf die Gründe für dieses ablehnende Verhalten hervorragender Gynäkologen eingehen, liegt es uns ob, diejenigen Operationsverfahren anzuführen, die in Deutschland an deren Stelle getreten sind. Und da ist es namentlich die ventrale Fixation des Uterus, welche diese Bedeutung hat und die zuerst von Köberlé bei Gelegenheit einer Castration im Jahro 1877 in der Weise ausgeführt wurde, dass der Uterus nach vorn gebracht und durch Einnähen der Stümpfe in den unteren Wundwinkel fixirt wurde. Während dieses Verfahren zuerst nur als Gelegenheitsoperation bei der extraperitonealen Stumpfbehandlung nach Ovariotomie angesehen wurde, machte im Jahre 1878 P. Müller den Vorschlag, bei hartnäckiger Retroflexio und Kuhn 1882 bei Prolaps, den Fundus uteri an die vordere Bauchwand anzuheilen, welchen Gedanken B. Schultze als sehr beachtenswerth bezeichnet, wenn die Laparotomie anderweitig indicirt sei. Olshausen, Czerny, Leopold, Sänger haben dann die Ventrofixation namentlich ausgebildet, so dass dieselbe immer mehr zum Selbstzwecke ausgeführt wurde. Czerny macht die Vernähung des Uteruskörpers, während Olshausen die Nähte nicht durch den Fundus uteri legt, sondern durch die Wurzeln der Ligamenta rotunda führt; beide wollen die Nähte nicht in die Bauchwunde, sondern seitlich von derselben in die Bauchwand gelegt wissen.

Allein die Empfindung, dass die Ventrofixatio uteri ein unverhältnissmässig grosser Eingriff sei im Hinblick auf die vollkommene Gefahrlosigkeit der damit behandelten Krankheit theilt offenbar nicht nur $\mathrm{Z}$ weifel, sondern sie hat wohl dazu geführt, dass die Indicationsstellung der Ventrofixation eine beschränkte geblieben 
ist. Fritsch drückt sich in dieser Beziehung aus: Sollten wir es erreichen, die Laparotomie zu einem ungefährlichen Eingriffe zu machen, so würde die Köberlé'sche Operation weiter studirt werden müssen; und auch Küstner betont, dass man es in dieser Beziehung mit der Laparotomie nicht ernst genug nehmen könne, indem eine solche neue Adhärenzen setzen kann, die gelegentlich schlimmere Folgen haben dürften, als die früheren Adhäsionen.

Um die Gefahren der Laparotomie zu umgehen, fixirten Asaky, Canera und Andere den Uterus an die Serosa der Banchdecken, ohne das Peritoneum zu eröffnen.

Später hat Schücking ein Verfahren angegeben, das darin besteht, einen Faden vom Mattermund aus dureh das Cavam uteri bis zum Fundus zu führen, von da aus das Corpus uteri und das vordere Scheidengewölbe zu durchsiechen und durch festes Anziehen des Fadens beim Knoten eine Befestigung des Fundus an der vorderen Scheidenwand zu bewirken, wodurch gleichzeitig eine künstliche Anteflexio zu Stande kommt. Dass bei dieser Methode, die Nadel ohne Controle in der unmittelbaren Nähe der Blase durchzuführen, Verletzungen der letzteren vorkommen, beweisen die Mittheilungen Zweifel's; auch Leopold sah einen Fall, in dem nach der Schücking'schen Operation in Folge Incrustation des Fadens Steinbildng eintrat.

Eine Verbesserung ist zweifellos die Zweifel'sche Modification des Schücking'schen Verfahrens: Zweifel zieht, um die Blasenverletzung zu vermeiden, nachdem er mit dem Paquelin einen Querschnitt in das vordere Scheidengewölbe gemacht hat, die Portio nach abwärts und drängt dann die Blase in die Höhe, worauf erst die Schücking'sche Vaginalfixation ausgeführt wird; eine Blasenverletzung ist auf diese Weise ausgeschlossen, wie dies sieben von Zweifel mit bestem Erfolge operirte Fälle beweisen, die Technik der Operation ist aber immerhin wohl eine etwas heikle Sache. Von Dührssen, Mackenrodt u. A. ist die Vaginaefixation weiter ausgebildet worden, so dass nun allerdings die Verletzung der Blase nicht mehr so sehr als Schreckgespenst im Vordergrunde steht. Die ursprüngliche Schücking'sche Operation dürfte zur Zeit wohl kaum mehr ausgeführt werden, indem bei derselben nicht nur die Blase, sondern gelegentlich auch eine Darmschlinge dūrchstochen, oder ein Ureter verletzt werden kann. Diese beiden letzteren Gefahren fallen bei den Vaginaefixationen nach Dührssen und Mackenrodt allerdings gänzlich fort; dass aber auch nach 
diesen Verfahren die Blase bei der Operation verletzt werden kann, habe ich in der allerletzten Zeit mehrfach direct und indirect in Erfahrung gebracht. $0 \mathrm{~b}$ es sich dabei, wie vielfach angenommen wird, immer um nachträgliche Einwanderung der Fäden in. die Blase handelt, ist mir mehr als zweifelhaft, indem ich selbst gesehen habe, wie von sehr geschickter Hand am SchultzeWinckel'schen Phantom ein Faden durch die Blasenwand durchgeführt worden war; ich gebe zwar zu, dass das secundäre Einwandern von Seidenfäden häufiger ist, indem eben bei Operationen in einem infectiösen Gebiete, wie es die Vagina vorstellt, keine Möglichkeit ist, die Seide aseptisch zu legen: und das Wandern von Seidenfäden führe ich auf Grund einer sehr grossen Erfahrung ausnahmslos auf infectiöse Processe zurück.

Sei dem nun wie ihm wolle: jedenfalls hat man mit der Möglichkeit zu rechnen, dass bei diesen Operationsverfahren einmal ein Fremdkörper früher oder später in die Blase gelangen und Cystitis oder Steinbildung zur Folge haben kann. Aus diesem Grunde würde ich der Mackenrodt'schen Methode vor allen Verfahren der Vaginaefixation den Vorzng geben, indem bei derselben die Fäden lang gelassen und nach 14 Tagen entfernt werden.

Andere in dieser Richtung zielende operative Eingriffe sind diejenigen ven Richelot, der die hintere Wand der Vaginalportion anfrischt und an die hintere Scheidenwand annäht, sowie die Verkürzung der Ligamenta sacro-uterina von Frommel, die in der Weise ausgeführt wird, dass der Uterus aus seinen Verwachsungen gelöst, nach vorn gezogen wird, worauf die beiden Ligamenta rectouterina in der Nähe ihres Abganges vom Uterus umstochen und am Peritoneum der seitlichen Beckenwand angenäht werden.

Wenn Löhlein auf Grund seiner Beobachtungen zu dem Schlusse kommt, dass, trotz der Fülle unseres therapeutischen Arsenals gegen dieselben, die Heilung der Retrodeviationen durch unsere üblichen Methoden die Ausnahme sei, so ist es sicher sehr gerechtfertigt, wenn wir auf Grund einer grösseren Reihe von wirklich ausgezeichneten Resultaten, die damit an der hiesigen Klinik erzielt worden sind, ein Verfahren dringend zur Nachprüfung empfehlen, das bei völliger Sicherheit und Eleganz der Ausführung die Wiederherstellung der physiologischen Verhältnisse erreicht: 
ich meine die Kocher'sche Modification der Alquié-Alexanderschen Operation.

Polk ist entschieden im Rechte, wenn er behauptet, den Ligamenta rotunda werde eine viel za geringe Wichtigkeit eingeräumt; denn unter normalen Verhältnissen reguliren die Ligamenta rectouterina, welche die Cervix nach hinten and die Lig. rotunda, die den Fundus uteri nach vorn halten, die Lage des Uterus.

Durch Anziehen der Ligamenta rotunde bewegt sich der Fundus uteri, wie man sich an der Leiche leicht überzeugen kann, vornüber -, aber nicht nur das, sondern durch energisches Anziehen und Verkürzen derselben kann auch ein prolabirter Oterus gehoben werden, vorausgesetzt, dass derselbe beweglich ist. Das Princip der Alquié'schen Operation, eine bilateral-symmetrische Suspension des Uterus an den Ligamenta rotunda beruht aIso entschieden auf einer mechanisch und physiologisch ganz richtig gedachten Voraussetzung. Um ihre Widerstandsfähigkeit gegen $\mathrm{Zug}$ zu erproben, hat Polk den Lig. rot. Gewichte angehängt und gefunden, dass das eine bei $4 \frac{1}{2}$, das andere erst bei 5 Pfd. riss und dass die Dehnung der Bänder erst beiner Belastung von $3 \mathrm{Pfd}$. begann. Tch selbst habe mehrfach die Tragkraft der runden Mutterbänder bestimmt und gefunden, dass die Polk'schen Angaben im Allgemeinen zu niedrig gegriffen sind. Von zwei allerdigs sehr kräftig entwickelten Ligamenten riss das eine bei einer Belastung von 10 Pfd. nach einer Minute, nachdem es sich bei einer Belastung ron 5 Pfd. in 2 Minuten von $8 \mathrm{~cm}$ auf $10 \mathrm{~cm}$ gedehnt hatte. Das andere, noch stärkere Band wurde in einer Länge von $12 \mathrm{~cm}$ isolirt und dehnte sich bei einer Belastung von $6 \mathrm{Pfd}$. bis auf $15 \mathrm{~cm}$, ohne zu zerreissen; erst bei einer Belastung von 11 Pfd. erfolgte die Ruptur nach 1/2 Minute, wobei Dehnung bis auf $16 \mathrm{~cm}$ stattgefunden hatte.

Ausserdem habe ich, um die Tragkraft der beiden Ligamente bei ihrem Zusammenwirken zu bestimmen, den Uterus an denselben aufgehängt und dann belastet. Erst nachdem allmälig eine Belastung bis auf 17 Pfd. stattgefunden hatte, trat Ruptur ein, allein nicht der Ligamente, sondern es wurde das eine Uterushorn abgerissen.

Das Alexander'sche Operationsverfahren nun besteht darin, dass in erster Linie die Ligamenta rotunda durch eine von der Spina ossis pubis ausgehende ca. $5 \mathrm{~cm}$ lange Incision parallel zum Ligamentum Pouparti aufgesucht werden. Man spaltet bis auf die 
Aponeurose des M. obliquus abdominis ext. und legt den Annulus inguinalis externus frei. Auf ein Fettklümpchen, an dem letzteres zu erkennen sei, macht Imlach speciell aufmerksam; übrigens ist der äussere Leistenring nach Spaltung der Haut ja immer leicht fühlbar. Das Ligament wird bei seinem Austritte aus dem Leistenring, oder an der Spina pubis aufgesucht und stumpf isolirt; worauf die Wunde antiseptisch gedeckt und die gleiche Procedur auf der anderen Seite ausgeführt wird. In einem 2. Acte wird der Uterus aufgerichtet und die Ligamente werden angezogen, bis eine merkliche Resistenz anzeigt, dass der Uterus aufgerichtet und durch genügenden Zug gespannt, festgehalten ist. Dann wird das Ligament mittels zweier Silberdrähte fixirt, welche die Haut und das Band fassen.

Adams legt einen dritten Faden durch das freie Ende des Ligamentes und den unteren Wundwinkel.

Die Wunde wird drainirt, die Kranke bleibt 3 Wochen lang im Bette und trägt in der ersten Zeit der Convalescenz ein Hodge'sches Pessar.

Gardener schlägt 1884 vor, die beiden Ligamente ünter dem Mons veneris zusammenzubinden.

Casati macht einen einzigen medianen Hautschnitt, der die beiden Leistenringe verbindet; dann kreuzt er die Ligamente und vernäht sic mit einander und dem Unterhautzellgewebe.

Segond verfährt wie Alexander; nur macht er Knopflöcher in die Fascie und verknüpft die Ligamente rings um die Pfeiler.

Schwartz kerbt den Leistenkanal ein, wenn der extrainguinale Theil der Ligamente zu schwaeh entwickelt ist, um einen kräftigen Zug zu ertragen; dann verkürzt er die beiden Bänder um 5-9 cm, legt wenigstens zwei Nähte durch die Preiler und das Ligament und lässt während 14 Tagen ein Pessar oder Tampon liegen.

Roux legt durch einen wenigstens $61_{2}-7 \mathrm{~cm}$ langen Hautschnitt den vordern Leistenring frei, ladet dessen ganzen Inhalt auf die Pincette, zieht die ganze Masse leicht an, worauf er nach Abdrängen des N. genito-cruralis, unter Zurückschieben des Peritonealkegels das Ligament vorzieht and gewöhnlich am ea. $10 \mathrm{~cm}$ verkürzt. Das freie Ende des Bandes wird an der Spina pubis angenäht und nun das ganze bis ins Innere des Leistenkanales hinein in der Weise fixirt, dass die Nadel jeweilen die beiden Pfeiler, dăs Ligament und die hintere Wand des Canales fasst. 
Roux verlässt sich auf die Festigkeit der Naht und nicht von jeder weiteren Pessarbehandlung ab.

In seinen ersten Fällen hat Kocher eine Modification der A lquiéschen Operation gemacht, welche sich an diejenige von Gardener anlehnt. Nach Isolirung der Bänder, energischem Anziehen derselben unter Zurückschieben des Peritonealkegels, wurde das eine Band subcutan unter der medianen Hautbrücke hindurch in die andere Wunde hinübergezogen und mit diesem Ligamente verknüpft, worauf die beiden vordern Leistenringe in der Weise geschlossen wurden, dass die Ligamente eingenäht wurden.

Schon in seinen ersten Fällen von Alexander-Operation hat Kocher indess, entgegen der von anderen Operateuren geübten Methode, die vordere Wand des Leistenkanales gespalten, um das Ligament möglichst central aufsuchen und fassen zu können.

Bei Ausführung der Operation an Leichen hat sich Kocher später überzengt, dass die Aufrichtung des Uterus viel prompter geschieht, wenn die Zugrichtung direct nach aussen gegen die Spina il. ant. sup. ausgeübt wird und er hat daher eine weitere Modification der Operation getroffen, die ich auf Grund einer Reihe sehr schöner Resultate, die Prof. Kocher damit erzielt hat, sowie auf Grund einer von mir mit vollem Erfolg vor einem Jahre ausgeführten Operation nicht warm genug empfehlen kann.

Kocher stimmt Roux ganz bei, wenn dieser sagt, man solle zur Auffindung der Ligamente gehörige Incisionen machen, nicht kleine Oeffnungen, denn in einem "Loche" habe man keine Aussicht, das runde Mutterband sicher zu finden. Er hält z. B. auch die Methode von Casati, der durch einen einzigen, nach oben concaven medianen Hautschnitt die beiden äusseren Leistenringe verbindet, nicht für glücklich, sondern macht auf jeder Seite, direct über dem Leistencanal von dem Annulus inguinalis externus beginnend, einen Schnitt bis an die Spina ant. sup. heran, wie zur Radicaloperation der Leistenhernien, wie zur Unterbindung der A. iliaca externa. Man trennt Haut und die stark ausgebildete Fascia superficialis, in welcher man die Vasa epigastrica superficialia zu. unterbinden bekommt, bis man auf die Aponeurose des M. obliquus abdominis externus gelangt ist. Nun macht er im Gegensatz zu dem bisherigen Vorgehen, auch einen gehörigen Schnitt durch diese Aponeurose und eröffnet auf diese Weise den Leistencanal. Roux trennt diese Aponeurose nicht und selbst die Fibrae intercolumnares nur ausnahmsweise. Es ist aber wesentlich, dass man das Lig. rotundum möglichst central 
Fig. 1

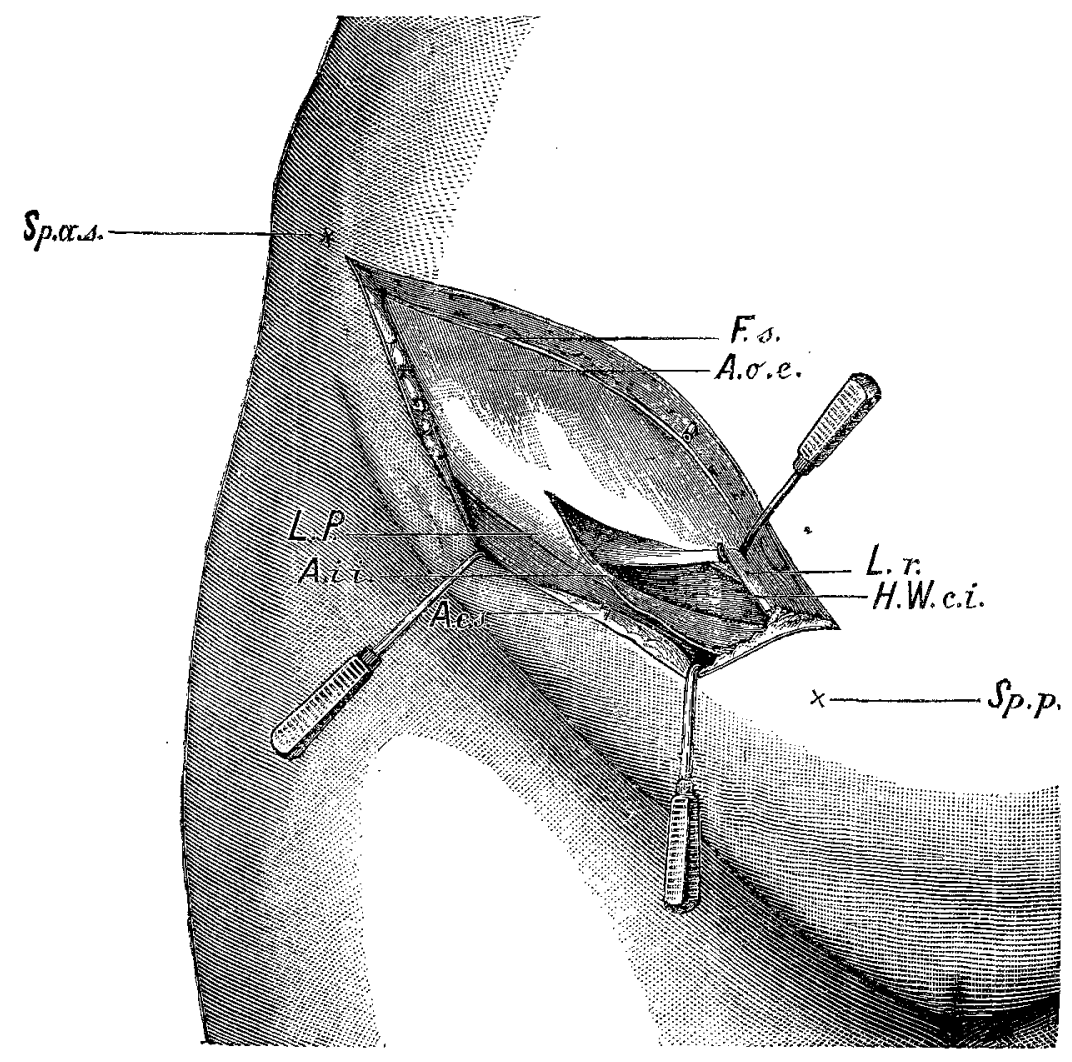

zeigt den klaffenden Schnitt dureh Haut, Unterhautfett mit A. e. s. = Arteria epigastrica und $E . s .=$ Fascia superficialis; derselbe reicht von $S p . \alpha_{.}$. . = Spina anterior superior ilei bis zur $S_{p \cdot p} \cdot=$ Spina pubis. Der Leistenkanal ist in ganzer Länge gespalten und der andere Rand des Schnittes durch seine Vorderwand $A . \bar{o} . e .=$ Aponeurosis musculi obliqui externi abwärts geklappt. Auf diese Weise wird die aufwärts schauende Rimne des $L . P$. = Ligamentum Pouparti freigemacht und das $L . r$. = Liganentum rotundun aus derselben emporgehoben mittels eines Hakens. So erscheint die H. W. c. $i$. = hintere Wand des Canalis inguinalis und über derselben am lateralen Eude der A.i.i. = Annulus inguinalis internus, aus welchem das Lig. rotundum herauskommt. 
aufsucht, damit man ganz sicher ist, dasselbe zu isoliren und an seiner ganzen Masse den gewünschten $\mathrm{Zug}$ ausüben zu können. Legt man das Band bloss an seinem Austritt aus dem vordern Leistenringe frei, so läuft man Gefahr, es bei etwas kräftigem Zuge zu zerreissen 1). Jetzt wird das Ligament emporgehoben, isolirt (Fig. 1), gegen die Symphyse zu von seinen peripheren Anheftungen frei gemacht und direct in der Richtung gegen die Spina ant. sup. ilei kräftig herausgezogen. Während ein Assistent die Stellung des Uterus per vaginam controlirt, werden die Ligamente nach beiden Seiten hin so gespannt, dass der Fundus uteri in normaler Höhe steht und nach beiden Seiten hin stramm gehalten ist. Das Vorziehen geschieht mit den Fingern, weil man so die Kraft am besten bemessen kann. Dabei wird mit dem Ligamentum rotundum ein $\mathbf{3 - 5} \mathrm{cm}$ langer Kegel des Parietalperitoneum mit emporgehoben. Derselbe wird von allenfalls mitfolgenden Weichtheilen möglichst genau isolirt und sobald man sich überzeugt hat, dass er leer ist und kein Eingeweide enthält, wird das Ligament auf der Aussenfläche des zwischen hinteren Leistenring und Spina ant. sup. ilei liegenden Theiles der Fascia obliqui externi durch eine fortlaufende Seidennaht festgenäht, welche auch den Peritonealkegel mitfasst. Auch die Naht erstreckt sich von der Spina ant. sup. ilei bis zum inneren Leistenringe herab. Dann wird durch tiefe Nähte durch die hintere Wand und die Schnittränder der Fascia obliqui externi der Leistencanal wieder geschlossen (Fig. 2). Als Nahtmaterial ist überall da, wo man eine feste Verklebung, wo man starke Verwachsungen, Adhäsionsbildung bezweckt, Seide, nie Catgut zu nehmen. Nach Schluss des Leistencanales wird die Hautwunde mit steriler physiologischer Kochsalzlösung irrigirt und durch fortlaufende Naht geschlossen.

Falls die Blutstillung eine völlig sichere ist, kann man auf jede Drainage verzichten und die Nahtlinien einfach mit einem sterilen Collodialstreifen decken; ist jedoch die Blutstillung nicht ganz gesichert, so thut man besser, die Wunden für 24 Stunden zu drainiren, weil sonst leicht ein Hämatom zu Stande kommt.

1) Wio mir erst nachträglich zur Kenntniss kommt, spaltet auch Edebohls in New-York (Arch. f. Gynäk. 1891. p. 168) den Leistenring und hebt als Vorzug des Verfahrens hervor, dass unter $26 \mathrm{zu}^{3 / 4}$ hervorgezogenen Ligamenten keines riss.

Die gleiche Methode befolgt nach einer Mittheilung in der Semaine médicalo rom 17. 9. $1892 \mathrm{Chalot}$. 
Fig. 2.

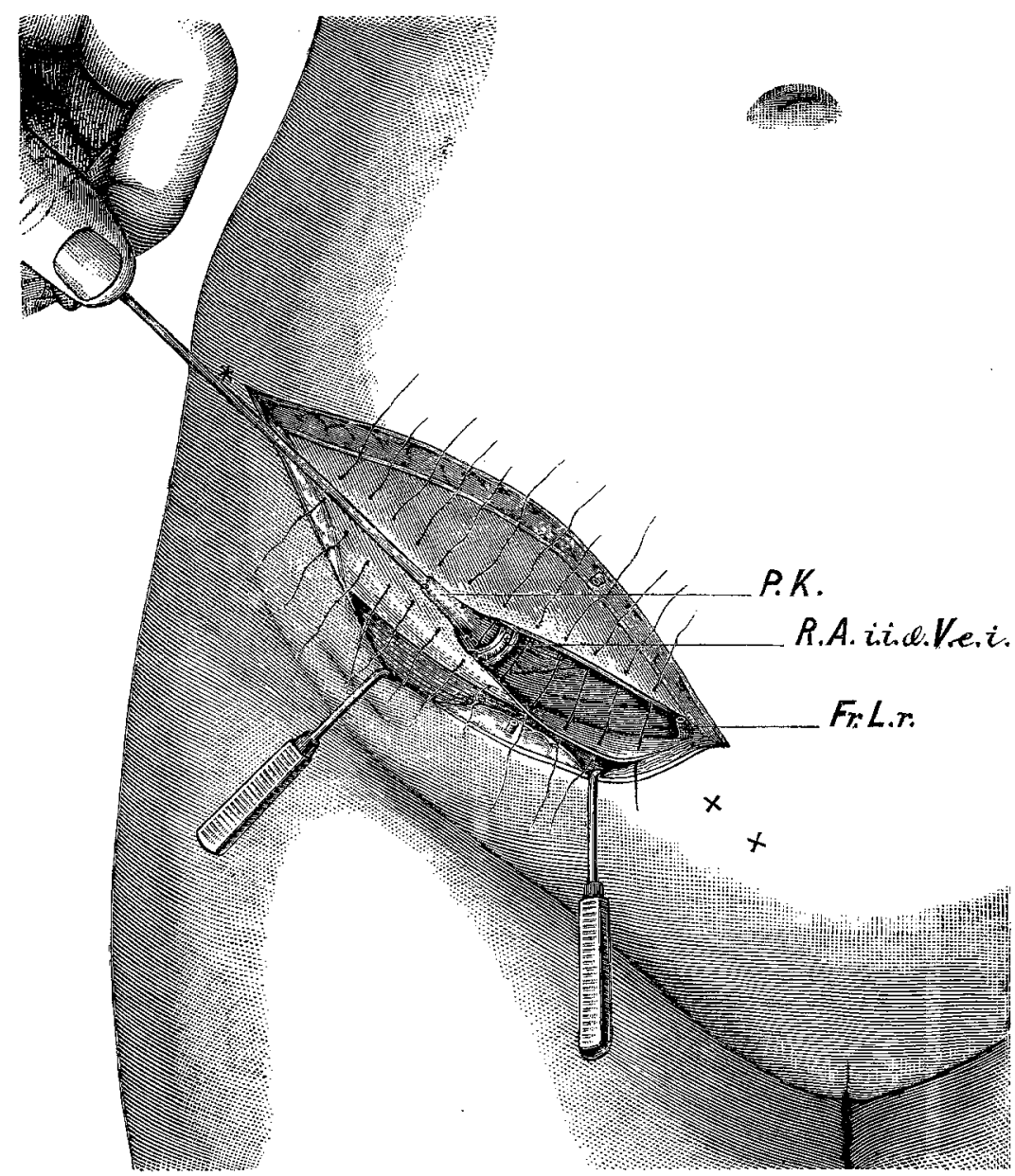

Das Lig. rotundum ist bei $T r . l . r .=$ Trennungsstelle des Ligamentum rotundum durchschnitten und unter kräftigem Herausziehen lateralwärts auf die Aponeurosis musculi obliqui externi umgeschlagen, um auf dieser durch multiple Nähte festgenäht zu werden bis zur Spina anterior superior ilei. Bei diesem kräftigen Vorziehen ist der $P . K .=$ Peritonealkegel, dessen Umschlagstelle deutlich zu sehen ist, mit herausgezogen (mehrere Centimeter lang). Derselbe wird ebenfalls in die Naht gefasst. Auf der hinteren Wand des Leistenkanals sieht man jetzt die Vasa epigastrica inferiora $=F$. e. $i$, entlang und parallel dem lateralwärts concaven Rande (R. a. i.i.) des Amnulus inguinalis internus verlaufend. 
Auf das Tragen eines Pessars nach der Operation verzichtet Kocher; er lässt die Kranken 14 Tage lang zu Bette liegen und empfiehlt ihnen bei der Entlassung aus dem Spitale, sich vorerst noch zu schonen.

Ich lasse unsere Krankengeschichten im Auszuge folgen.

Die 5 ersten Fälle hat Kocher nach seiner alten Methode operirt.

Fall 1. Frau R., 49 J., verheirathet.

Anamnese: 33jährig heirathete Pat. und kam 10 Monate später nieder, wobei ein starker Dammriss zustande kam. Schon während des Wochenbettes prolabirte der Uterus und fiel beim Aufstehen und nach einer Anstrengung jedes Mal vor. Ein Ring brachte keine Abhülfe und eine Bandage wurde nicht gut vertragen. Der Uterus liegt seit 2 Jahren ständig total vor. Muttermundslippen stark geschwollen und ulcerirt. Hochgradige Cystocele, während eine Rectocele fehlt. Der Damm ist nur etwa $1 \mathrm{~cm}$ breit, die Scheide ausserordentlich weit.

Operation (10. 7. 1888): Sublimatantisepsis. Incision parallel dem Lig. Pouparti auf beiden Seiten über dem äusseren Leistenring. Die Ligamenta rotunda werden freigelegt, vorgezogen und vom Processus vaginalis peritonei, der sich mit herausstülpt, getrennt. Nun wird das linke Band durch das subcutane Fett nach der rechten Wunde hinüber gezogen und mit dem rechten verknüpft. Die beiden Leistenkanäle werden durch Knopfnähte mit Seide geschlossen und es werden die Ligamenta an der Bruchpforte durch Knopfnähte ebenfalls fixirt. Dann wird die Wunde durch tiefe und fortlaufende Naht geschlossen, nicht drainirt und nur mit einem Collodiumstreifen bedeckt.

Verlauf: Entfernung sämmtlicher Nähte am Tage nach der Operation und Erneuerung des Collodiumastreifens. Am 20. 7. steht die Patientin mit sehr schön p. p. geschlossener Nahtlinie auf. Das unmittelbare Resultat der Operation ist ein vorzügliches, indem der Finger in der ganzen Länge eingeführt werden muss, um den Muttermund zu fühlen. Auch beim Stehen und Pressen zeigt sich keine Neigung zu Prolapsus nteri, dagegen besteht die Tasche in der vorderen Vaginalwand noch. Es wird daher noch eine Colporraphia ant. et post. mit Erfolg ausgeführt.

Laut schriftlichem Bericht vom 24. 9. 1888 ist der Prolaps der vorderen Vaginalwand nicht völlig zurückgegangen; dagegen ist der Uterus nicht mehr vorgefallen und die Patientin ,fühlt sich manchen Tag recht woh], wie sie es seit vielen Jahren schon nicht mehr war." Laut schriftlichem Bericht vom 21.9. 1892 ist der Uterus später wieder prolabirt, jednch nicht in dem Maasse wie früher, und tritt nur zeitweilig vor. Zudem soll der Prolaps in den letzten Jahren nicht zugenommen haben, so dass Pat, angiebt, „durch diese Operation Erleichterung bekommen zu haben". Es ist nicht sowohl der Prolaps, als die Cystocele, die ihr Beschwerden macht.

Fall 2. Fran S., 31 J., verheirathet.

Dammriss bei der ersten Entbindung. Nach der dritten Niederkunft Prolapsus, weshalb Pat. einen Ring trägt, der aber mannigfache Beschwerden verursacht. 
Die rechte Niere steigt bei tiefer Inspiration herunter, so dass man sie leicht fassen kann; dieselbe ist etwas druckempfindlich.

Nach Entfernung des Ringes erweist sich der Uteruskörper als ausgesprochen druckempfindlich, vergrössert und retroflectirt. Druck auf die Blasengegend erregt Urindrang"; Urin trübe, reagirt indess sauer und ist frei von Eiweiss.

Operation (12. 11. 1888): Keilförmige Excision der erodirten Portio. Dann wird die Alexauder'sche Operation angeschlossen. Es wird zuerst rechts der vordere Leistenring freigelegt. Man findet indess kein Lig. rotundum und erst nachdem der Canal nach oben aufgeschlitzt worden ist, sieht man ein Gebilde, dessen Natur erst unsicher ist, sich aber beim Anziehen als Ligamentum rotundum anfweist, indem es nach und nach sehr dick wird und den Processus vaginalis peritonei mit sich zieht. Derselbe wird zurückgeschoben, wodurch das Band isolirt wird. In gleicher Weise wird auf der linken Seite verfahren. In Folge der Erfahrung, die man rechts gemacht hat, wird der Leistenkanal links gleich aufgeschlitzt und das Band gefunden. Jetzt wird das linke Band subcutan in die rechte Wunde hinübergezogen und mit dem rechten verknüpft; der Knoten wird durch eine darüber gesetzte Seidenligatur gehalten. Dann werden die Leistenkanäle durch Naht geschlossen und ein Theil jedes Bandes wird damit eingenäht; tiefe und fortlaufende Hautnaht, keine Drainage.

Verlauf: Entfernung der Nähte am 14. 11; Collodiumstreifen. Vom 22. 11. steht Pat. auf und wird, nachdem die Periode vorüber ist, zur Perineoplastik wegen eines nicht unbedeutenden Dammdefectes geschritten. Von der Mitte der hinteren Vaginalwand, 2-3 $\mathrm{cm}$ unterhalb der Portio, wird ein Schnitt nach dem unteren Ende jeder kleinen Lippe geführt, dann längs der grossen Labien bis zum hinteren Ende des Risses nach dem Anus zu. Der dadurch gebildete Schleimhautlappen wird excidirt und es wird in der gewöhnlichen Weise zusammengenäht. Die Pat. wird am 19. Tage entlassen. Die Lage des Uterus ist eine sehr gute.

In einem schriftlichen Berichte vom 16. 4. heisst es von der Frau: „elle se porte admirablement maintenant" und am 30.4.89 stellt sich die Pat. selbst wieder vor. Sie hat ein gutes Aussehen. Die Narben sind sehr schön lineär und leicht beweglich. Die Portio vaginalis steht so hoch, dass, während die Mitte der Grundphalanx des Zeigefingers an den Schambogen stösst, die Spitze des Fingers die vordere Muttermundlippe erreicht. Der etwas nach rechts abweichende Fundus uteri steht 3 Querfinger unterhalb des Nabels in normaler Anteflexion. Der Damm ist gut gebildet. Druck auf die Blasengegend ist nicht empfindlich und die Urinbeschwerden haben abgenommen.

Dem Briefe eines namhaften Chirurgen, der Gelegenheit fand, die Patientin am 8. August 1890 zu untersuchen, entnehme ich die Stelle: "Ich habe das Vergnügen, Thnen mitzutheilen, dass mir das Resultat der Operation ein vollkommen gelungenes zu sein scheint: Uterus an normaler Stelle, in leichter Anteflexion, mässig vergrössert. Pat. fühlt sich durch die Operation ausserordentlich erleichtert. - " Das Gleiche besagt ein Brief der Patientin vom 22. 9. 1892.

Fall 3. M. K., 34 J., verheirathet.

Hat 3 Mal geboren. Nach der letzten Geburt längere Erkrankung im Wochenbett und ein zunehmender Vorfall der Gebärmutter, der sie 
daran hindert, stehende Arbeit zu verrichten. Ein Versuch, den Prolaps mit Pessarien zurückzuhalten, missglückte.

Bei der inneren Untersuchung findet man den Muttermund beinahe im Niveau der Vulva. Wenn sich Pat. anstrengt, treten Portio und invertirte Vaginalwände ca. $3 \mathrm{~cm}$ vor die Vulva. Die Scheide ist sehr weit und schlaff; der Uteruskörper ist als sehr beweglicher, eigrosser, kugeliger, derber Körper über der Portio zu fühlen, lässt sich mit Leichtigkeit über das Promontorium hinaufschieben. Die Scheide erstreckt sich in Folge alter Dammrisse bis zum Anus.

Operation (9. 1. 1889): Freilegung der Lig. rotunda, wie in den ersten Fällen, Rechts reisst das Peritoneum dabei ein, wird durch eine Ligatur geschlossen. Die von ihren terminalen Ansătzen befreiten Ligamente werden nun zusammengeknüpft in der Weise, dass das eine unter der Hautbrücke vor den Musculi recti durchgestossen und mit den anderen verknotet wird; der Knoten wird mit einer Seidenligatur gesichert. Der Uterus, welcher vor der Operation in der Scheide sichtbar war, ist so hoch hinanfgerutscht, dass er mit dem Finger nach der Operation kaum mehr erreichbar war.

Pat. wird am 28. 1. entlassen. Die Portio befindet sich in Rückenlage und im Stehen in normaler Höhe.

Allein schon drei Monate später trat die Frau, die 8 Tage nach ihrer Entlassung ihre Arbeit (stehend an einem hohen Tische in einer Schuhfabrik) wieder aufgenommen hatte, mit einem Recidive ein, das sich in den letzten Tagen bemerkbar machte. Am 20. 4. wurde die Colporrhaphia anterior und posterior mit Perineoplastik ausgeführt.

Die Patientin verliess am 6. 5. 1889 das Spital mit völlig reponirtem Prolaps, hat sich seither nicht wieder vorgestellt und kann auch schriftlich nicht erreicht werden.

Fall 4. I. F., 49 J, verheirathet.

Eine Entbindung vor 6 Jahren, wobei ein Dammriss entstand. Allmälige Entwickelung eines Prolapsus. Der Dammriss reicht fast bis zur Analöffnung, Prolapsus beider Scheidenwände. Uterus tief stehend, retroflectirt.

Operation (16. 1. 1889): Wie oben. Beim Zuge an den runden Mutterbändern folgt das Peritoneum sofort als spitzer Kegel und wird zum Theil zurückgeschoben, zum Theil an der Basis noch zugebunden, um keinen Bruchsack zu bekommen. Auf der linken Seite zeigt sich ein Bruchsack, der neben dem herausgezogenen Peritonealkegel liegt. Er wird isolirt und abgetragen. Dann werden die Ligamente in der rechten Wunde in der geschilderten Weise zusammengeknotet und der Leistenkanal und die Wunde durch fortlaufende Seidennaht geschlossen.

Patientin wird am 18. 2, entlassen. Der Uterus ist antevertirt, im vorderen Scheidengewölbe deutlich fühlbar, beweglich. Die Portio steht $6 \frac{1}{2} \mathrm{~cm}$ über dem Arcus pubis, ist leicht nach hinten gerichtet. Pat. klagt noch über häufigen Urindrang.

Am 3. 4. zeigt sich die Frau wieder. Der Uterns ist in guter Stellung, antevertirt. Dagegen ist die vordere Wand der Scheide stark prolabirt. Cystocele vaginalis. Colporrhaphia anterior. Patientin wird 29. 4. 1889 geheilt entlassen, hat sich nicht wieder vorgestellt und ist unauffindbar. 
Fall 5. Frau E. S., 55 J., verheirathet.

Im Anschluss an ihre zweite Niederkunft vor 24 Jahren ein Vorfall, der bis vor 2 Jahren ohne Beschwerden ertragen wurde. Dann wiederholte Operationen seitens eines Gynäkologen. Anlegung eines Ringes, der aber seinen Zweck nur nnvollkommen erfüllt. Hochgradiger Prolapsus uteri.

Operation (28. 3. 1889): Wie oben. Die beiden Bänder sind so stark angezogen worden, bis der controlirende Assistent angiebt, den Mattermund mit dem Finger kaum mehr zu erreichen.

Am 18. 4. wird die Frau entlassen mit kaum sichtbaren Narben. Bei Rückenlage der Frau muss der Zeigefinger in seiner ganzen Lünge eingeführt werden, um die Portio zu fühlen. Beim Untersuchen im Stehen muss man den Zeigefinger bis zur Mitte der Grundphalanx einführen, um die Portio zu erreichen. Die Portio ist leicht nach hinten gerichtet und steht auf der hinteren Vaginalwand.

Im Mai 1889 findet sie sich in der Schamspalte, daher Amputatio portionis und Dammbildung nach Lawson-Tait. Im December 1889 ist der Prolapsus noch nicht wieder eingetreten. Die Vulva klafft; vom Uterus ist nichts zu sehen. Sein unteres Ende steht beim Liegen in normaler Höhe; auch beim Stehen und bei starkem Pressen tritt der Uterus nicht bis zum Damm herunter, doch wölbt sich dabei die vordere Vaginalwand kugelig vor.

Pat. hat sich seit dem 23. 12. 1889 nicht wieder vorgestellt; ist zur Zeit unbekannten Aufenthalts.

\section{Die weiter folgenden Fälle sind nach der neuen Modification} von Prof. Kocher mit directem Seitenzuge operirt worden.

Fall 1. Fran V. D., 63 J., verheirathet.

3 Jahre nach der letzten Geburt entstand ein Vorfall, der 13 Jahre lang, bis 1881, mit Hysterophor behandelt wurde. Da erkrankte sie an einer Bauchfellentzündung, wegen welcher sie fast ein Jahr lang liegen musste. Als sie wieder aufstand, stellte sich ein Scheidenvorfall ein und eingelegte Hysterophore nützten nichts mehr. Namentlich wegen hochgradiger Urinbeschwerden unterwarf sie sich im November 1882 einer plastischen Operation und trug daraufhin wieder einen Ring. Andamernde Beschwerden veranlassten Pat, von Neuem operative Abhülfe zu suchen. Sehr weite Vagina und starke Kolpitis. Die Portio steht nach vorn, das Corpus uteri von etwas derber Consistenz, im hinteren Scheidengewölbe fühlbar. Beim Pressen nähert sich der Uterus dem Scheideneingang, ohne dass er ganz zwischen die Labien heraustritt.

Operation (31. 7. 1889): Freilegung der Ligamenta rotunda auf beiden Seiten mit Spaltung der Haut parallel zum Ligamentum Pouparti. Da Prof. Kocher sich an der Leiche überzeugt hat, dass der Effect der Operation besser ist, wenn die Ligamente nach aussen gezogen werden, wurden die Leistenkanäle gespalten und die Ligamente nach Ablösung von der Spina pubis durch einen Fascienschlitz in der Nähe der Spina anterior sup. hindurchgezogen und hier, nachdem sie stark angezogen worden sind, ein erstes Mal fixirt. Dann wird unter doppeltem Mitfassen des Ligaments der Leistenkanal wie bei der Radicaloperation geschlossen. Der während der Operation durch einen Assistenten controlirte Effect ist ein sehr guter. Die Wunden. werden durch fortlaufende Naht ohne Drainage geschlossen. 
Verlauf: Ein kleiner Fadenabscess schliesst sich erst nach 2 Monaten nach Abstossung mehrerer Ligaturen.

Am 23. 8. wird eine Perineoplastik nach Lawson-Tait ansgeführt, wodurch man einen sehr hohen und breiten Damm bekommt.

Am 8. 10. geheilt entlassen.

Laut schriftlichem Berichte vom 27.9.1892 ist der Erfolg der Operation ein anhaltender gewesen, die Patientin frei von Beschwerden.

\section{Fall 2. Fran M. S., 37 J., verheirathet.}

Nach dem ersten Wochenbette vor 9 Jahren, stellte sich ein Vorfall der Scheidenwände und schliesslich der unteren Hälfte des Uterus ein. Nach vielfachen vergeblichen Versuchen, mit Pessarien auszukommen, wünschte Patientin operative Behandlung.

Beim Stehen liegt die Portio vor den Genitalien. Die vordere Vaginalwand ist ganz vorgefallen, hinten dagegen kann der Finger in einer Länge von etwa $6 \mathrm{~cm}$ eingeführt werden. Der Uterus lässt sich gut zurückbringen; die Vulva ist klaffend, der Damm etwas schlaff, doch nicht besonders kurz.

Operation: (30. 12. 1889.) Anf beiden Seiten wird der vordere Leistenring freigelegt und der Leistencanal durch Spaltung der Fascie des M. obliquus abdom. ext. aufgeschnitten. Das Ligamentum rotundum wird stumpf ausgelöst, nachdem die dasselbe von aussen kreuzenden Vasa epigastrica inferiora unterbunden worden sind. Die beiden Bänder werden peripherwärts abgeschnitten und kräftig nach der Spina ant. sup. hingezogen. Dabei wird ein ca. $3 \mathrm{~cm}$ hoher Conus des Peritoneum herausgezogen. Da der mit dem Finger per vaginam controlirende Assistent angiebt, dass die Hebung des Uterus noch keine vollständige ist, wird beschlossen, das Peritoneum zu eröffnen. Das sich vordrängende Netz wird reponirt und der jetzt am Ligamentum rotundum ausgeübte $\mathrm{Zug}$ erweist sich als viel wirksamer. Der nun controlirte Erfolg ist der, dass der Uterus gut emporgezogen und fest eingespannt ist, dagegen ist der Prolaps der vorderen Scheidenwand nicht zurückgegangen. Die Peritonealscheide wird wie ein Bruchsack durchstochen, ligirt und abgetragen; das Ligament unter kräftigem Anziehen gegen die Spina ant. sup. hin durch Knopfnähte, die das Peritoneum und oben und unten die Ränder der durchschnittenen Fascia abdom. ext. mitfassen, angeheftet. Prof. Kocher hebt während der Operation den Vortheil dieser Mlethode als darin bestehend hervor, dass seröse Flächen mit einander in Contact gebracht werden und dadurch eine feste Verlöthung erzielt wird. Die Wunden werden fortlanfend genäht, nicht drainirt, mit Collodialstreifen gedeckt.

Patientin verlässt das Spital am 18.1.90.

Man fühlt den Uteruskörper 2 Querfinger über der Symphyse rechts von der Mittellinie. Derselbe lässt sich etwas herunterdrücken, nimmt aber sofort wieder seinen Platz ein. Nach oben lässt er sich etwa $2 \mathrm{~cm}$ weit hinaufschieben. Die Seitenverschiebung ist gering. Die vordere Vaginalwand ist vorgewölbt, ohne zu prolabiren. Im Stehen bleibt der Uterus in der angegebenen Höhe; nur wölbt sich die vordere Vaginalwand mehr vor.

Eine spätere Untersuchung (13, 3. 1890) ergiebt, dass der Fundus uteri stark 2 Querfinger über der Symphyse deatlich fühlbar, etwas nach rechts liegt. Die Portio vaginalis steht etwa $5-6 \mathrm{~cm}$ hoch über der Vulva. Beim 
Untersuchen im Stehen, muss man die $\mathbf{2}$ ersten Phalangen des Zeigefingers einfübren, um die Portio zu erreichen. Patientin hat sich seit der Operation andauernd vorzüglich gut befunden. Der Prolaps ist nie zum Vorschein gekommen. Die Menses sind regelmässig.

Ein ebenso befriedigender Erfolg wird am 22. IX. 1892 constatirt.

3. Fall. M. C., 36 J., verheirathet.

Seit der 2. Niederkunft vor etwa 14 Jahren Prolapsus uteri. Nachdem 2 Jahre ein Pessarium getragen, traten erst vor einem Jahre neue Beschwerden seitens des Vorfalls ein.

Da mehrere Versuche, mit Pessarien den Uterus zurückzuhalten, fehlschlugen, wünscht Pat. die Operation.

Operation: (2. 4. 1890) wie oben. Die schlecht entwickelten Ligamente sind in der dicken subserösen Fettschicht schwer zu finden. Nach Spaltung des Leistenkanales werden sie kräftig in der Richtung der Spina ant sup. ilei angezogen, so dass ein $4-5 \mathrm{~cm}$ langer Peritonealkegel mitgezogen wird. Der per vaginam controlirende Assistent constatirt, dass die Portio $7 \mathrm{~cm}$ über der Symphyse gefühlt wird. Dann wird das Ligament sammt Peritonealkegel, immer unter energischem Anziehen bis zur Spina ant. sup. herauf an die Fascien durch Knopfnähte fixirt, darauf sein peripheres Stück in der umgekehrten Richtung zurückgeklappt und durch Knopfnähte, die gleichzeitig den Leistencanal in sehr vollkommener Weise schliessen, noch einmal befestigt.

Die Heilung wird durch einen kleinen Fadenabscess verzögert.

Bei der Entlassung der Patientin (17. 7.) steht der Uteruskörper in der Mitte zwischen Nabel und Symphyse und lässt sich nur wenig herunterdrängen.

2 Monate später derselbe Befund.

4. Fall. Frau M. B., 60 J., verheirathet.

Pat. hat eine Cruralhernie, die schon mehrmals, eben jetzt wieder, eingeklemmt gewesen ist. Daneben besteht seit $30 \mathrm{Jahren}$ in Folge ihrer ersten und einzigen Geburt ein Gebärmuttervorfall, welcher indess, abgesehen von häufigem Urindrang, nicht sonderliche Beschwerden bereitet.

Erscheinungen alter Lues (Knochenauftreibungen, Perforation des weichen Gaumens, multiple Drüsenschwellungen). Rechtsseitige, leichte reponible Cruralhernie. Die Portio reicht beim Stehen ins Nivean des Scheideneinganges; auch beim Pressen fällt der Uterus zur Zeit nicht weiter vor.

Nachdem Pat. eine Jodkalikur durchgemacht hat, wird am 2. 7. zuerst die Radicaloperation ihrer rechtsseitigen Cruralhernie vorgenommen, dann die Fascie des M. obliquus abd. ext. hart am oberen Rande des Ligamentum Pouparti eingeschnitten, dann das mühelos gefundene weissliche, strangförmig runde Ligament stumpf freigelegt und mit dem bedeckenden Peritonealkegel kräftig nach aussen und oben gezogen. Der Assistent, der den Stand des Uterus prüft, giebt an, dass bei einseitigem Zuge der erreichte Erfolg nur ungefähr $1 \mathrm{~cm}$ beträgt, während bei doppelseitigem Zuge sofort $3 \mathrm{~cm}$ erreicht werden. Unter stetigem Zuge nach aussen oben wird nun das Band durch Seidenknopfnähte bis zur Spina ant. sup. herauf in den Eascienschnitt eingenäht, wobei auch das Peritoneum mitgefasst wird. Das periphere Ende des Bandes wird rückwärts angelegt und durch tiefgreifende Nähte, die zu gleicher 
Zeit die Bruchpforte schliessen, noch einmal fixirt bis beinahe zu der Spina pubis herunter.

Auf der linken Seite bildet sich ein Abscess, so dass erst nach 14 Tagen die Wunde durch Secundärnaht geschlossen werden kann.

Bei der Entlassung (17.8.91) steht der Fundus uteri 3 Querfinger über der Symphyse; auch beim Drängen und Husten tritt derselbe nicht herunter. Keine Schmerzen bei der Untersuchung. Die vordere Vaginalwand stark gewölbt, aber nicht vorgefallen.

Pat. ist im August 1892 an Carcinoma pylori gestorben. Ihr Arzt (Dr. Haas in Muri) fand bei der Section den Fundus uteri in normaler Lage hochstehend, nach beiden Seiten hin festgehalten.

5. Fall. Frau S. M., 58 J., verheirathet.

Pat. will seit 25 Jahren an einem Gebärmuttervorfall leiden, den sie vor 2 Jahren hat operiren lassen; es wurde eine plastische Operation gemacht und seither soll die Gebärmutter nie mehr ausgetreten, die Beschwerden nicht mehr so bedentend gewesen sein. Im Juni 1890 will sich Pat. durch einen Fall eine linksseitige -Leistenhernie zugezogen haben, die schon öfters leichtere Einklemmungserscheinungen gesetzt hat.

Die Uvula fehlt vollständig, der weiche Ganmen ist kurz und zeigt narbige Veränderungen. In der rechten Leistenbeuge wölbt sich bei Anspannung der Bauchdecken eine wallnussgrosse Geschwulst vor mit tympanitischem Percussionsschall; das Lig. Pouparti ist unterhalb derselben fühlbar. Per vaginam fühlt man in Rückenlage die vergrösserte Portio $4 \mathrm{~cm}$ hinter dem Scheideneingang; beim Stehen tritt dieselbe ins Niveau der Schamspalte herunter. Die vordere Vaginalwand ist mässig vorgefallen.

Operation (16. 12. 1891): Radicaloperation der rechtsseitigen Leistenhernie, indem ein Schnitt von der Spina pubis bis zur Spina ant. sup. ilei geführt wird, der gleichzeitig dazu dienen soll, das Ligamentum rotundum freizulegen und zu befestigen. Der Bruchsack wird isolirt, eröffnet, möglichst central durchstochen, ligirt und entfernt, worauf das Ligamentum rotundum an der Spina pubis abgeschnitten und energisch gegen die Spina ant. sup. hingezogen wird, so dass sich der Uterus ganz bedeutend hebt und nach der rechten Seite hinüberneigt. Mit dem Bande wird ein ca. $4 \mathrm{~cm}$ langer Conus seines Peritonealüberzuges vorgezogen und sammt dem Ligamente in die Pfeiler eingenäht; letzteres wird bis an die Spina ant. sup. hinan mit ca. 12-15 Seidenknopfnähten in der Weise befestigt, dass dadurch zugleich der Leistenkanal geschlossen wird. In gleicher Weise wird mit dem linken Ligamente verfahren und dasselbe nach Zurückdrängen des Peritonealkegels so angezogen, dass der Uterus aufgerichtet in normaler Höhe steht. Drainage der beiden Wunden mit Glasdrains; tiefe und fortlaufende Naht. Verband mit steriler Gaze.

Am 30. 12. steht Pat. zum ersten Male auf und wird am 13.1.92 entlassen. Dabei findet Prof. Kocher, dass man Zeige- und Mittelfinger tief in die Vagina einführen muss, um an die Portio zu gelangen. Dieselbe steht in der Mitte. Die obere Hand fühlt das Promontorium deutlich; in dem horizontalen Niveau desselben steht der Fundus uteri, der auch beim Stehen und starken Pressen nicht tiefer heruntertritt.

Am 20. 9. 1892 findet man das Orificium ext. $10 \mathrm{~cm}$ über 
dem Scheideneingange. Beim Pressen tritt die Portio nicht tiefer - also ein sehr befriedigendes Resultat. Die Cystocele vaginalis, die schon vor der Operation bestand, ist durch dieselbe nicht gehoben worden, verursacht aber der Patientin kaum Beschwerden. Rechts, wo zugleich die Radicaloperation der Hernie ausgeführt wurde, ist nicht die geringste Andeutung einer solchen vorhanden; links dagegen tritt beim Pressen eine hühnereigrosse Netzhernie auf der Höhe des hinteren Leistenringes vor. Die Patientin wird gleich der Radicaloperation unterworfen und nach einigen Tagen p. p. geheilt aus dem Spital entlassen.

Der folgende Fall von totalem Prolaps mit Inversion der Scheide, Elongation und Hypertrophie des Collum wurde als Controlfall operirt, indem Prof. Kocher an demselben nur den Wirkungsgrad seiner Methode bei einem so ausgeprägten Falle feststellen wollte und von vornherein in Aussicht nahm, durch spätere plastische Operationen nachzuhelfen.

6. Fall. Fran M. H., 44 J., verheirathet.

Hat 5 Mal geboren. Nach der 2. Niederkunft Scheidenvorfall. Seit der letzten Entbindung hat der Vorfall immer mehr zugenommen.

Aus der Vagina ragt eine faustgrosse, von normaler Schleimhaut bedeckte Geschwulst hervor, an deren unterem Ende auf der hinteren Fläche das Orificium ext. uteri als quergestellte Oeffnung sichtbar ist. Die vordere Vaginalwand ist ganz vorgefallen, der Fornix verstrichen. Die hintere Wand ist nicht ganz prolabirt, der Finger kann hier ca. $3 \mathrm{~cm}$ eindringen. Cystocele und Rectocele.

Die Vaginalwände sind sehr schlaff und hypertrophisch. Die vordere Wand ist stets prolabirt in der Schamspalte sichtbar. Introitus vaginae sehr weit; Damm $3 \mathrm{~cm}$ breit.

Operation (16.2.92) wie oben. Das rechte Lig. rotundum wird peripher abgeschnitten, isolirt und seitwärts nach der Spina ant. sup. so kräftig angezogen, dass ein Peritonealkegel mitfolgt und der Uterus bis zum Vestibulum aufwärts ohne Nachhülfe bewegt. Das Ligament wird unter beständigem Zuge durch eine fortlanfende Naht an die Fascia abdom. ext. befestigt, dann sein distales Ende medialwärts zurückgeschlagen und nun noch einmal in seiner ganzen Länge der Fascie eingenäht, wobei die Bruchpforte zugleich geschlossen wird. Ein kleiner Bruchsack, der sich vorfindet, wird isolirt, unterbunden und abgetragen.

Die gleiche Operation wird auf der linken Seite ausgeführt. Der Zug am Bande wirkt auch auf der linken Seite sehr gut und es steht nun der Uterus ganz hoch und nach beiden Seiten hin festgehalten. Die Wunden werden ohne Drainage fortlaufend genäht und mit steriler Gaze verbunden.

Am 16. 3. wird Patientin entlassen. Prof. Kocher findet, dass in Rückenlage die Portio nur mit ganz eingeführtem Finger zu fühlen ist. Beim Drängen wölbt sich die vordere Scheidenwand stark vor, kommt aber nicht in die Vulva herein. Der Stand des Uterus wechselt nicht wesentlich beim Stehen, nur wölbt sich die vordere Vaginalwand vor.

Am 21. 4. 92 findet Prof. Kocher beide Leistennarben etwas eingezogen. Der Prolapsus vaginae hat sich nicht wesentlich verschlimmert. 
Der Finger muss im Stehen $6 \mathrm{~cm}$ weit eingeführt werden, um an die Portio zu gelangen. Beim Husten und Pressen tritt jedoch die Portio tiefer, bleibt aber immerhin noch ca. $4 \mathrm{~cm}$ hinter der Schamspalte. Der Fundus uteri ist 3 Querfinger über der Symphyse füblbar.

Bei einer Untersuchung am 25. 9. wird eine wesentliche Verschlimmerung des Prolapsus vaginae beobachtet. Auch der stark elongirte Cervix ist tiefer getreten, so dass der Patientin die Colporrhaphie und Amputation der Portio, die wie oben gesagt, von vorn herein in Aussicht genommen war, vorgeschlagen wird. Vorläufig kann sich die Frau jedoch nicht dazu entschliessen, weil die vor der Operation bestehenden Beschwerden bis jetzt gänzlich verschwunden sind.

7. Fall. Fräulein F., 31 Jahre.

Vor 8 Jahren wurde wegen Retroflexio ein Pessar eingeführt, das aber nichts nützte. Anch später eingelegte Intrauterin- und Hebelpessarien erfüllten ihren Zweck nicht. Bei der Defäcation empfindet Patientin dentlich ein mechanisches Hinderniss im Rectum.

Descensus uteri, mit Retroversionsstellung, so dass der Uterus, der etwas vergrössert ist, ganz nach hinten und die Portio nach vorne gerichtet ist. Beide Ovarien sind deutlich zu fühlen; das linke ist ausgesprochen druckempfindlich.

Operation am 23. 3. 92 wie oben. Die Ligamente werden sofort gefunden, isolirt und mit einem Peritonealkegel, der etwas zurückgeschoben wird, etwa $6 \mathrm{~cm}$ weit vorgezogen. Dann werden sie mässig gespannt gegen die Spina ant. sup. hin durch Seidenknopfnähte fixirt, wobei zugleich die Bruchpforte geschlossen wird. Die Untersuchung nach der Operation ergiebt vollkommene Rectification der Retroversion und Hebung der Portio um $5-6 \mathrm{~cm}$.

Patientin wird am 25. 4. entlassen. Der Uterus steht normal, dementsprechend hat die Patientin auch keine Beschwerden mehr.

Eine Untersuchung im August 1892 constatirte, dass der Erfolg der Operation ein dauernder geblieben ist. Der Zeigefinger muss in ganzer Länge eingeführt werden, um an die Portio zu gelangen. Der Fundus nteri ist 2 Querfinger über der Symphyse fühlbar. Beim Untersuchen im Stehen, tritt die Portio beim Pressen etwas tiefer, doch behält der Uterus seine normale Anteflexionsstellung vollkommen. Laut schriftlicher Nachricht vom September 1892 ist das Resultat ein gleich befriedigendes geblieben.

Ausser diesen Fällen sind von Prof. Kocher in letater Zeit noch 3 Patientinnen der Alexander-Operation unterworfen worden; die beiden Fälle kommen jedoch für die Frage der Dauerheilung nicht in Betracht.

Als Epikrise zu den mitgetheilten Krankengeschichten will ich nur darauf hinweisen, wie gewaltig der Unterschied zwischen der ersten und der zweiten Operationsserie ist. Während bei der eigentlichen Alexander'schen Operation die Resultate in den ersten 5 Fällen sehr mangelhafte waren, hat in den 7 nach der Kocher'schen Methode operirten Fällen das Resultat ausnahmslos 
der Erwartung entsprochen, die man von dem Eingriffe erhofft hatte.

In der Revue médicale de la suisse romande vom 20. November 1888 hat Prof. Roux in Lausanne eine sehr bemerkenswerthe Arbeit über die Alexander'sche Operation für Retroflexio und Prolapsus uteri veröffentlicht, in welcher in sehr zutreffender Weise unter Mittheilung von 8 eigenen, mit sehr befriedigendem Resultate operirten Fällen, die Gründe des zeitweiligen Misslingens der Operation und die Aussichten auf besseren Erfolg derselben erörtert werden. Es darf nach der eben mitgetheilten Casuistik auch wohl auffallen und ist im Hinblick auf die schönen Resultate Kocher's kaum zu begreifen, dass diese ,singulière idée" von Alquié in Montpellier, die schon im Jahre 1840 concipirt worden ist, noch jetzt auf so grossen Widerstand stösst. Ausser dem von Roux angeführten Grunde, dass eine Reihe hervorragender deutscher Gynäkologen eigene Operationsmethoden erfunden haben, dürfte doch sicherlich die vielfach mangelhafte Austührung der Operation Schuld an dem abfälligen Urtheile über dieselbe sein. Dazu kommt, dass die Regio inguinalis viel mehr den eigentlichen Chirurgen als den Gynäkologen ein vertrautes Operationsfeld ist, während letztere sich mehr auf die vaginalen Methoden zur Behandlung des Leidens beschränken. Es dürfte aber ein grosser Vortheil sein, aus dem zweifelhaften Gebiete vaginaler Antisepsis sich durch die Alexander'sche Operation wieder an eine Stelle emporgehoben zu sehen, wo man klar und sicher eine aseptische Wundbehandlung durchführen kann, zudem diese saubere und leichte Operation viel mehr leistet, als ihr bis jetzt zugestanden wird. Prof. Roux und Dr. Haas haben Gelegenheit gehabt, den Erfolg der Alexander-Operation durch Autopsie zu controliren. Sechs Monate nach der Operation, welche wegen hartnäckiger Retroflexion and Retroversion unternommen worden, fand Roux bei der anatomischen Untersuchung den vergrösserten Uterus in normaler Lage, den Fundus leicht anteflectirt, gegen die hintere Blasenwand lehnend. Er liess sich nach hinten drängen bis zur Berührung mit dem Promontorium und nahm spontan wieder die normale Lage ein, welche er beibehielt nach völliger Entleerung der Blase. Die Ligamenta rotunda vom Uterus bis zum hintern Leistenring maassen rechts $4-4 \frac{1}{2} \mathrm{~cm}$, links $5 \frac{1}{2}-6 \mathrm{~cm}$. Das Peritoneum zeigte an dieser Stelle keine Ausstülpung, vielmehr normale Verschieblichkeit. Das Ligament bildete ein gänsekieldickes Bündel, 
war mit der Umgebung in- und ausserhalb des Leistenkanales verwachsen. Aehnliche Verhältnisse scheinen in dem von uns erwähnten Falle, der ein Jahr nach der Operation zur Autopsie kam, vorgelegen zu haben.

Wenn wir nun auf die Einwände, die gegen die Operation erhoben worden und auf die Punkte, die zur Erzielung eines genügenden und bleibenden Erfolges namentlich zu berücksichtigen sind, genauer eintreten wollen, so wäre vielleicht folgendes zu bemerken:

1. Die Schwierigkeit der Operation ist sicher allgemein überschätzt worden. So halten z. B. Halliday Croom, Skene, Keith $\dot{u}$. A. die Auffindung des Bandes für ausserordentlich schwierig und zeitraubend. Es ist aber entschieden die Orientirung über die Spina pubis und den vorderen Leistenring und damit auch die Auffindung des Ligamentes, wie ich aus eigener Erfahrung am Lebenden und an Leichen weiss, von grösster Leichtigkeit. Ganz wesentlich erleichtert die Spaltung des Leistenkanals, die mehr centrale Isolirung des Ligaments die Operation; immerhin stimme ich Beurnier bei, welcher das Einüben der Operation für erforderlich hält. Auch Mundé meint, dass es in allen Fällen leicht gelingt, die Ligamenta rotunda zu finden, er sieht die Ursache, weshalb Andere und er selbst früher öfters an dem Versuche scheiterten, darin, dass die Angaben Alexander's allzu minutiös befolgt wurden.

2. Allan's Angabe, dass die Ligamente bei alten Frauen einer fettigen Degeneration unterliegen, habe ich in mehreren Fällen nicht bewahrheitet gefunden; an der Leiche einer 70 jährigen, sehr fettleibigen Person habe ich ganz gat entwickelte Lig. rot. gefunden. Derselbe Autor hebt nach Versuchen an der Leiche hervor, dass die Operation unmöglich sei, wenn die Ligamente zu dünn, zu stark fixirt oder zu brüchig seien. Sind aber die Bänder wirklich einmal ausnahmsweise schwach entwickelt, so braucht man sie ja nur so weit central nach dem Uterus hin zu verfolgen, bis sie die nöthige Sicherheit bieten, dass ein gehöriger Zug ertragen wird.

3. Das Bedenken späterer Hernienbildung ist wenigstens, wenn man nach Kocher operirt, durchaus ungerechtfertigt, indem ja der ganze Leistenkanal geschlossen wird und die Operation ganz die gleiche Effectwirkung haben muss, wie die Bassini'sche Radicaloperation der Leistenhernien. Findet man bei der Operation einen wirklichen Bruchsack, so isolirt man denselben genau, zieht 
ihn energiseh vor, worauf man den Bruchsackhals durchsticht, abbindet und den Bruchsack entfernt. Die Peritonealausstülpung, welche dem Ligament bei kräftigem Anziehen stets nachfolgt, kann man stumpf mit einem Gazebäuschchen (cf. Fall 5 der zweiten Serie), zurückwischen, oder, was vorzuziehen ist, den Peritonealkegel mit dem Ligament in den Inguinalkanal einnähen; die Furcht, dadurch die Vitalität des Ligamentes zu schädigen, ist ungerechtfertigt.

4. Die Gefahr der Operation ist bei aseptischem Vorgehen null; sie ist durchaus nicht grösser als diejenige einer gewöhnlichen Incision und jedenfalls bei weitem nicht so bedeutend, wie diejenige, welche die neueren Verfahren nach Schücking, Frommel etc., sowie die Laparotomie setzt. Roux betont ganz bezeichnend: "tous les dangers sont le fait de l'opérateur et non de l'opération en elle-même."

5. Der Vorwurf, der immer wieder gegen die Operation erhoben wird, und von dem natürlich ihre Berechtigung völlig abhängt, ist der ihrer geringen Leistungsfähigkeit. Die vielfach mangelhafte Ausführung und die Application der Methode auf ungeeignete Fälle sind unserer Erfahrung nach die beiden Hauptfactoren, die das abfällige Urtheil vieler Autoren erklärlich machen. Richelot hat ganz Recht, wenn er betont, dass eben nicht nur die schlechte Stellung, sondern auch die übrige Beschaffenheit des Organes zu berücksichtigen sei.

Es sind ferner vor Allem 2 Categorien streng auseinanderzuhalten: die beweglichen und die fixirten Retrodeviationen; für letztere, sowie für complicirte Prolapsus uteri wird man mit der Alexander'schen Operation allein allerdings niemals ein befriedigendes Resultat erzielen. In den 2 einzigen Fällen, wo Polk Misserfolge zu verzeichnen hatte, lag fixirte Retroflexion vor, bei welchem Zustande ja Alexander selbst die Operation für contraindicirt hält. Polk schliesst sich auf Grund seiner Erfahrungen dem Postulate Alexander's an, dass man nur operiren solle, wenn man den Uterus mit der Sonde aufrichten kann. Auch Adam's erste Operation ist ihm vollständig misslungen, da der Uterus durch Adhäsionen fixirt war. Für solche Fälle ist eine orthopädische Vorbehandlung nöthig oder die Laparotomie zu machen.

Was die Ausführung der Operation anbelangt, so haben wir uns sehon darüber geäussert: man muss eben nicht nur pro forma 
die Ligamente verkürzen, sondern dieselben langsam aber kräftig herausziehen, bis sich der Uterus so weit nach vorn und oben dislocirt, dass der Fundus in strammer Suspension unmittelbar hinter und über der Symphyse steht. Wenn sich Sänger äussert: „Die immer noch gegen die Alquié-Alexander'sche Operation ablehnende Haltung der deutschen Gynäkologen ist nicht freundlicher geworden, da die bisherigen Resultate der keineswegs ungefährlichen Operation sehr viel zu wünschen übrig lassen" und die Methode auf Grund einer eigenen Beobachtung verurtheilt, so ist ein solches Urtheil unserer Ansicht nach nicht gerechtfertigt, indem Sänger, um das Verfahren kennen zu lernen, einen einzigen Fall nach Alexander operirt, und dazu noch auf der einen Seite das Band nicht gefunden hat! Seine Operation ist also nicht vollständig ausgeführt worden und darf nicht als Beweis gegen die Operation als solche ins Feld geführt werden. Sänger's fernere Behauptung: „Die ventrale Fixation des retroflectirten Uterus, sei es nach der älteren, oder nach der neueren, rationelleren und verlässlicheren Methode von Olshausen ist das bislang einzige, direct operative Verfahren, welches den Uterus in Anteversionsstellung zu erhalten geeignet ist", bedarf also einer Correctur zu Gunsten Alexander's, die Kocher'sche Modification derselben vorausgesetzt. Es schützt übrigens sogar die Ventrofixation, wie wir sehen werden, nicht vor Recidiven, was Fälle von Klotz u. A. beweisen. Wenn Sänger dafür die Unzulänglichkeit der Befestigungsart beschuldigt, so bedienen wir uns mit dem gleichen Rechte der gleichen Waffe und erklären die mangelhafte Ausführung der Methode für die Misserfolge der Alexander-Operation verantwortlich.

Fälle wie diejenigen von Skene Keith, wo die Wunden fünf Wochen brauchten, um zu heilen, sind ebenso wenig beweisend für den Werth oder Unwerth der Operation, wie diejenigen, wo das Ligament nur einseitig gefunden oder nur pro forma etwas verkürzt wurde; denn die Infection kann sehr wohl Gangrän der Ligamente zur Folge haben und damit fällt die Fixation derselben dahin.

Auch hier möchte ich wieder betonen, dass das Kocher'sche Operations-Verfahren des directen seitlichen Zuges nach aussen hin, d. h. in der Richtung des im Abdomen liegenden Antheils des Bandes und der Befestigung des Ligamentes in derselben Richtung nach der Spina ant. sup. hinauf und zwar in ganzer Länge desselben die Aussichten der Dauerheilung bedeutend vermehrt. Ich 
habe mich bei Controle per vaginam während der Operation and am Cadaver oft überzengt, wie schön der Uterus bei beiderseitigem Zuge an den Ligamenten nach aussen emporgezogen und der Fundus nach vorn gebogen, „wie durch zwei Zügel" aufgerichtet wird. In einem sehr ausgesprochenen Falle von Retroflexio uteri an der Leiche einer alten Frau, wo der im Uebrigen normale Uterus ganz nach hinten abgeknickt im Douglas'schen Raume lag, gelang mir die Aufrichtung allerdings zuerst auch nicht; allein, sowie die beiden Bänder in einer Ausdehnung von ca. $10 \mathrm{~cm}$ vorgezogen und nun ganz central an ihren Peritonealkegeln gefasst wurden, gelang sie ohne manuelle Nachhülfe vòn unten ganz plötzlich. Dabei riss das Peritoneum auf der einen Seite ein, was bei energischem Zuge auch am Lebenden $a b$ und zu vorkommen wird, bei sicherer Asepsis jedoch absolut bedeutungslos ist. Wir heben hervor, dass oft viele Centimeter lange (6, $8 \mathrm{~cm}$ und mehr) Stücke des vorgezogenen Ligaments abgeschnitten werden, zum Beweis, wie kräftig das Band vorgezogen wird. Um den Werth der Operation gerecht beurtheilen zu können, müssen wir natürlich die Arten der Lageveränderung, die zu dem Eingriffe Veranlassung gaben, streng auseinanderhalten: es ist eben nicht gleichgültig, ob die Verkürzung der Ligamenta rotunda für eine mobile oder fixirte Retrodeviation, ob sie für einen einfachen Prolaps oder für einen solchen, der mit Cystocele-Rectocele, mit secundären Veränderungen des Uterus complicirt ist, ausgeführt wird. Die unsichere Indicationsstellung, die mangelhafte Auswahl der geeigneten Fälle ist es, welche die Alexander'sche Operation vielerorts in Misscredit gebracht haben; diese, sowi die angeführten Factoren, die bei der Operationstechnik in Betracht kommen, erklären es auch, weshalb die Ansichten über den Werth des Verfahrens einander scheinbar so unversöhnlich entgegen gesetzt sind. Es verhält sich mit der Alquiéschen Operation eben ganz ähnlich, wie mit der Radicaloperation der Hernien: die Schuld des Misserfolges liegt in den meisten Fällen nicht an der Operation als solcher, sondern sie fällt dem Operateur zur Last. Wie kann man denn hierüber noch im Zweifel sein, wenn Mundé, abgesehen von seinen 4 ersten Fällen, später. $19 \mathrm{Mal}$ mit constantem Erfolge operirte bis auf einen Fall, wo die Verhältnisse besonders ungünstig lagen. Alexander führt schon 188526 Fälle von Retroversio und Retroflexio an, die nach seiner Methode operirt wurden, mit constantem Erfolg; ebenso haben Trélat, Reid, Terrillon, Berruti, Gardener in je 5 Fällen 
von mobiler Retroversion ohne einen einzigen Misserfolg operirt. Schwartz hat von 10 Fällen 3, Strong von 6 Fällen 2 Misserfolge bei adhärenten Retroversionen und Casati kommt auf Grund einer Zusammenstellung von 139 Fällen verschiedener Autoren zu dem Schlusse, dass die Alexander-Operation eine Radicaloperation für nicht complicirte Retroflexion sei. Bei Anlass später aus anderweitigen Ursachen ausgeführter Laparotomieen haben sich Polk u. A. überzengt, dass der Uterus die durch den operativen Eingriff erlangte Lage vollständig beibehalten hatte. Nach dem gleichen Autor hat die Operation ebenso wenig ungünstigen Erfolg auf die Function der Blase, wie auf eine später auftretende Schwangerschaft und es sind in der That mehrere Fälle bekannt von Dauerheilung nach Jahren, welche die Prüfung durch neue Graviditäten hindureh siegreich bestanden.

Während wir also bei Retrodeviation die richtig modificirte Alexander'sche Operation für eine vorzügliche Behandlungsmethode halten, geht, was die Anwendung unserer Operation bei Prolapsus uteri anbelangt, aus den eben mitgetheilten Kocherschen Fällen mit Sicherheit hervor, dass sie auch bei nicht complicirten Vorfällen die grösste Berechtigung verdient. Bei Prolaps im Anfangsstadium genügt entschieden die Kocher'sche Modification der Alexanderoperation allein; falls auch die Vagina vorgefallen ist, müssen die üblichen plastischen Operationen angeschlossen werden. Polk hat bei Prolaps $3 \mathrm{Mal}$ nach Alexander mit rollkommenem Erfolge operirt, ebenso Sinclair, Allan u. A. Gardener hat zwei sehr schöne Resultate aufzuweisen neben einem unvollkommenen, bei welchem aber das Ligament nur um einen Zoll verkürzt wurde; Matlakowski, der die Ligamente sehr energisch vorzieht und sie um $8--10 \mathrm{~cm}$ kürzt, hat in 3 Fällen von Prolaps die Operation ausgeführt und bei später wegen Rectocele nöthig gewordenen Kolporrhaphieen gefunden, dass der Uterus hoch stand wie nach der Operation. Doléris schlägt für den Prolaps vor, die äblichen plastischen Methoden mit der Alexander'schen Operation zu combiniren. Er verwirft das Vorgehen Alexander's, zuerst die Verkürzung der Ligamenta rotunda und dann erst die Cystocelenoperation vorzunehmen; er will zuerst die Cystocelenoperation und erst in zweiter Linie Alexander's Operation und bei letzterer nur das eine Ligament verkürzt wissen, damit die Ausdehnung der Blase eine ungehinderte sei. Was den ersten Punkt anbelangt, kann man ihm allenfalls beistimmen, doch glaube 
ich nicht, dass die Gründe, die or dafür angiebt, stichhaltig seien, und es scheint mir natürlicher, zuerst zu versuchen, wie viel man mit der Alexander'schen Operation erreicht; allfällig nöthige plastische Operationen können dann immer noch als Nachoperationen hinzugefügt werden. Der zweite Vorschlag von Doléris ist aber entschieden zu verwerfen, weil die Verkürzung eines Ligamentes eben nicht genügt. Nur wenn man von vornherein wegen ausgesprochener Cysto- oder Rectocele plastische Operationen in Aussicht nimmt, dann mag es von Vortheil sein, mit diesen zu beginnen, um nicht später durch das Herunterziehen die verkürzten Ligamente wieder zu dehnen.

Wenn wir nun das Verfahren der ventralen Fixation, das wenigstens unter den deutschen Gynäkologen der AlquiéAlexander'schen Operation den Rang völlig abgelaufen hat, einer kurzen Kritik unterziehen wollen, so müssen wir vorausschicken, dass uns aus der Kocher'schen Klinik keine eigenen Erfahrungen zu Gebote stehen, sondern dass wir unsere Schlüsse lediglich auf Grund der über diese Frage vorliegenden Literatur ziehen.

Das Misslichste an der Ventrofixation ist der Umstand, dass dieselbe die Laparotomie involvirt, denn wenn auch die directen Gefahren derselben durch die Asepsis auf ein Minimum reducirt sind, so ist sie immerhin doch kein bedeutungsloser Eingriff. Es liegen sodann Erfahrungen vor, dass die Bildung einer künstlichen Spalte zwischen Uterus und Blase schon mehrfach zu inneren Darmeinklemmungen Anlass gegeben hat. Ferner ist die Operation deshalb nicht ideal, weil sie aus einer pathologischen Lage eine neue pathologische Lage schaffe, indem sie den normal beweglichen Uterus fixirt. Und was schliesslich den schwerwiegendsten Grund gegen die Ventrofixation abgiebt, ist die Beobachtung, dass sogar sie nicht vor Recidiven sichert. So hat P. Müller ,in einer Reihe von 12 Fällen, wo entweder andere Verfahren im Stiche liessen (unter denen jedoch Alexander's Operation nicht in Anwendung kam), oder die Untersuchung eine so hochgradige Erweiterung und Erschlaffung der Geschlechtstheile ergab, dass voraussichtlich Kolporrhaphieen nicht zum Ziele geführt hätten, die ventrale Fixation mit und ohne Abtragung des Uteruskörpers vorgenommen. Der Erfolg war jedoch keineswegs den Erwartungen entsprechend. Nur in ganz wenig Fällen wurde hierdurch die Lageverändereng beseitigt. In einigen Fällen verlängerte sich der angeheftete Cervix so beträchtlich, dass auf diese Weise wieder Prolapsus vaginae 
eintrat. Das Gleiche kam auch auf die Weise zu Stande, dass die Befestigungsstelle in die Bauchwand trichterförmig eingezogen wurde. Auch vollständige Loslösung des Gebärmutterstumpfes mit vollständjger Wiederherstellung des Vorfalles wurde beobachtet.“ Freund, Mundé, Hugo, Hofmeier u. A. haben mit der Ventrofixation ähnliche schlimme Erfahrungen gemacht, während allerdings Czerny, Leopold, Sänger u. A. mit ihren Resultaten zufrieden sind. Olshausen betrachtet die blosse Einnähung des Geschwulststieles in die Bauchwunde nach Exstirpation eines Tumors meist als nicht genügend, um die Lage des Uterus dauernd zu rectificiren. Er fixirt die beiden Uterushörner an die Bauchdecken in der Weise, dass er die dem Uterus benachbarten Theile der Ligamenta rotunda und lata, ihre Wurzeln anheftet; trotzdem hatte er bei seinen zwei ersten Ventrofixationen einen Misserfolg und sagt daher: „nur in sehr vereinzelten und sonst trostlosen Krankheitsfällen von adhärenter Retroflexio oder Prolapsus, glaube ich, dass die Operation am Platze sein kann." Aehnlich sprechen sich Küstner, Frommel, Hegar, Ziegenspeck u. A. aus. Dass allerdings auch nach einer gelungenen Ventrofixation der Verlauf der Gravidität ein völlig normaler sein kann, bewoisen Beobachtungen von Sänger, Klotz, Leopold und Kümmel; ja auf Grund von Erfahrungen Sänger's ist es nicht einmal wahrscheinlich, dass irgend welche Erschwerung oder Störung der Schwangerschaft eintritt.

Kümmel hat unter 9 nach Alexander operirten Fällen nur etwa in der Hälfte Dauerheilung erzielt, trotzdem er die Ligamente möglichst weit nach dem Uterus hin verfolgte und spricht sich deshalb gegen die Operation aus. 'In 6 Fällen von fixirter Retroflexion erreichte er durch Laparotomie und Ventrofixation dauernden Erfolg, indem er die misslichen Erfahrungen Müller's, sowie Freund's, der die Ventrofixatio wegen Prolapsus oder Retroflexio nur als Gelegenheitsoperation ausführte, aber niemals Erfolg davon sah, dadurch zu entgehen suchte, dass er den Fundus uteri ans Periost der Symphyse fixirte. Es würde uns ausserordentlich freuen, wenn Kümmel durch die Kocher'schen Resultate veranlasst, Versuche mit dessen Methode machen würde.

Die Alexander-Operation, die sich darauf beschränkt, den Uterus wieder in seine normale Lage zurückzubringen, hat vor der ventralen Fixation jedenfalls neben ihrer völligen Gefahrlosigkeit den Vortheil voraus, dass der Uterus gegen seine Umgebung be- 
weglich bleibt. Wir möchten daher in Anbetracht der eben mitgetheilten Casuistik die Indicationen zur Ventrofixation möglichst beschränkt wissen und sie begrenzen auf die irreponiblen, fixirten Rückwärtsbeugungen und recidiven Prolaps, wo die Beschwerden den bedeutenden Eingriff rechtfertigen. Bei bestehenden Verwachsungen wäre immer zuerst noch zu versuchen, den Uterus nach Thure Brandt beweglich zu machen und dann nach Alexander aufzurichten und bei Prolapsus vaginae wäre die Verkürzung der Lig. rot. mit plastischen Operationen zu verbinden. Nur wenn aus anderer Ursache laparotomirt wird, dann kommt die ventrale Fixation in erster Linie in Frage; sie wird also meist nur als Gelegenheitsoperation in Verwendung kommen.

Wenn wir nun unsere Meinung über den Werth der Alexanderschen Operation noch einmal präcisiren wollen, so kommen wir zu dem Schlasse, dass für mobile Retrodeviationen und nicht complicirten Prolaps die Operation wenigstens nach der Kocher'schen Modification die Grundbedingungen eines operativen Eingriffes erfüllt: bleibende Beseitigung des Leidens und Herstellung eines dem physiologischen sich nähernden Zustandes bei absoluter Gefahrlosigkeit. Aber auch für complicirten Prolaps ist AlexanderKocher eine stets schätzenswerthe Hülfsoperation. Die Operation ist in Misscredit gerathen, weil sie zu oft als blosse Scheinoperation ausgeführt worden ist; es ist für das Gelingen derselben von fundamentaler Bedeutung, dass man die Ligamenta rotunda, nachdem man dieselben durch Spaltung der vorderen Wand des Leistencanales central aufgesucht hat, genau isolirt und kräftig und weit vorzieht. Wie man das dabei in einem langen Kegel sich ausziehende Peritoneum behandelt und mitbenutzt, haben wir genau geschildert. Diese beiden Forderungen geiten für das Ligamentum rotundum ebenso gut wie für den Bruchsack bei der Radicaloperation der Hernien, wenn diese wenigstens die Bezeichnung ,radical" verdienen soll.

Ebenso gut wie für die gewöhnlichen Hernien nicht das lästige Bruchband, sondern die Radicaloperation, ebenso gut ist für bewegliche Retrodeviationen nicht das widerliche Pessar, sondern die Alquié-Alexander'sche Operation das Normalverfahren.

In welcher Weise die Kocher'sche Modification des ursprünglichen Verfahrens dessen unmittelbaren bleibenden Erfolg zu sichern geeignet ist, glauben wir in Obigem zur Genüge belegt zu haben. 
Uebrigens kann sich Jeder, der die Controle an der Leiche machen will, von der unmittelbaren Wirkung der Kocher'schen Methode mit Leichtigkeit überzeugen.

So weit es mir möglich ist, in der Frage der operativen Behandlung der Retrodeviationen und des Prolapsus uteri eine Stellung einzunehmen, glaube ich, dass die Zukunft entweder der Alexander-Kocher'schen Operation oder der Vaginalfixation - unter Nachhülfe mit entsprechenden plastischen Operationen gehören wird, da für die grosse Mehrzahl der Fälle die Laparotomie von vornherein gar nicht in Frage kommt.

Die Entscheidung, welches Verfahren schliesslich den Sieg davon trägt, wird auf Grund eines statistisch verwerthbaren Materiales ausfallen, das die nächsten Jahre - bei dem regen Interesse für diese Specialfrage - voraussichtlich liefern werden. Möge diese Entscheidung ohne Voreingenommenheit gefällt und nicht ausschliesslich der einen oder anderen Richtung gehuldigt werden. Auch die "Altera pars" gegenüber der momentan herrschenden Strömung zu ihrem Rechte gelangen zu lassen und für die AlquiéAlexander'sche Operátion eine Lanze einzulegen, war der Zweck dieser Arbeit.

\section{Literatur.}

K ocher, Th. Chirurg. Operationslebre. Fischer. Jena 1891.

Alquié. Sur une nouvelle méthode etc. (Bulletin de l'académie de médicine f. VI. 1840, cit. nach Pozzi.)

Alexander, W. Behandlung von Retroflexion und Prolapsus uteri durch die neue Methode des Verkürzens der Ligamenta rotunda. London. Churchill 1884 (ref. Centralbl. f. Gynäk. 1885, No, 7. pag. 109).

Alexander. British gynecol.-journ. Nop. 1885 (cit. nach Pozzi).

Allan. Alexander's Operation (Lancet 1884, 7. Juni, ref. Centralbl. f. Gynäk. 1885. No. 7. p. 111).

Assaky. Ueber extraperitoneale Hysteropexie. Arch. für Gynäk. 18891. pag. 152.

Bournier. Untersuchungen über die Ligamenta rotunda des Uterus and deren Verkürzung durch die Alexander'sche Operation (Gaz. des hôpitaux 1888. No. 2 and 4; ref. Centralbl. f. Gynäk. 1889. No. 14. pag. 238).

Casati, E. Modification der Alexander'schen Operation (Raccoglitore med. 1887. No. 5-8; ref. Centralbl. f. Gynäk. 1888. No. 26). 
Crespi, G. L'isteropexia extraperitoneale nella cura degli spostamenti posteriori dell' utero (Gaz. degli ospit. 1890; ref. Centralbl. f. Chirurgie 1891. No. 5).

Czerny, V. Deber die Vernähung der rüokwärts gelagerten Gebärmutter. (Beitr. zur klin. Chirurgie. Bd. IV. Heft 1.)

Doléris. Operative Behandlung des Uterusprolapses duroh Kolpoperineorrhaphie und Verkürzung der Ligamenta rotunda. (Nouv. arch. d'obstétr. et de gynécol. 1886. No. 6; ref. Centralbl. f. Gynäk. 1887. No. 23).

Doléris. Le raccourcissement des ligaments ronds. Nouvelles arohives d'obstétrique. Paris 1887.

Doléris et Riecard. (Union médicale. No. 85.)

Freund, H. W. Berioht über die Verhandlungen der gynäkologischen Sektion der 62. Versammlung deutsoher Naturforscher und Aerzte za Heidelberg. 1889. (Centralbl. f. Gynäk. 1889. No. 43.

Frommel, R. Ueber operativo Behandlung des retroflectirten Uterus. (Centralbl. f. Gynäk. 1890. No. 6. pag. 94.

Gardener, W. Alexander-Adam's Operation. (Centralbl. für Gynälr. No. 7.)

Kümme1, H. Zur Fixation des Uterus. Deutsche Medicinalzeitung 1892. No. 49.

Küstner. Die Entstehungsbedingungen der Retroversio-flexio und des Prolapsus. (Zeitschr. f. Geburtsh. u. Gynäk. Bd. XI., Heft 2; ref. Centralbl. f. Gynäk.)

Küstner, O. Die Behandlung complicirter Retroflexionen und Prolapse etc. Samml. klin. Vortr. Septbr. 1890.

Leopold. Ueber die Annähung der retroflectirten Gebärmutter an der vorderen Bauchwand. (Volkmann'sche Sammlung No. 333 und Centralbl. f. Gynäk. 1888. No. 11.)

Mackenrodt, A. Die operative Behandlung der Retroflexio uteri. Beschreibung eines neuen Verfahrens. Deutsche med. Wochenschrift-1892. No. 22.

Malgaigne, S. F. Introduction dans les oeurres d'Ambroise Paré: Sur l'origine et les progrès de la chirurgie etc. Paris 1840.

Matlakowski. Alexander's Operation bei Prolapsus uteri. Ref. Centralblatt f. Gynäk. 1888. No. 39. p. 641.

Müller, P. Ueber Exstirpation des Uterus (Corresp.-Bl. f. Schweiz. Aerzte 1878. No. 20 und 21).

Mundé, P. Der Werth von Alexander's Operation (Amer. Journ. of Obstetr. Octbr. 1888. Ref. Centralbl. f. Gynäk.).

Olshausen, R. Ueber ventrale Operation bei Prolapsus und Retroversio uteri (Centralbl. f. Gynäk. 1886. No. 43).

Jlshausen. Centralbl. f. Gynäk. 1885. No. 43.

?ozzi, S. Lehrbuch der klin. und operativen Gynäkologie. Uebers. von Dr. Ringier 1892.

?raeger, S. Zur Ventrofixatio uteri. Centralbl. f. Gynäk. 1891. No. 16.

'iqué, I. Ueber ventrale Fixation des Uterus nach Olshausen wegen Retroflexio (Bull. gén. de thérap. 1889. Jan. Ref. Centralbl. f. Gynäk. 1889. No. 28). 
Polk, W. M. Alexander's Operation. New-York med. record 1886. Ref. Centralbl, f. Cirurgie. 1887. No. 47).

Reid, W. L. Drei Fälle von Alexander-Adam's Operation (Glasgow med. journ. Nov, 84. Ref. Centralbl. f. Gynäkol. 1885. No. 7).

Rossier, G. Ueber die Kolporrhaphia anterior duplex (Archiv f. Gynäk. Bd. XXXVIII. Heft 8).

Richelot. Union médicale 1868 . No. 58 and 59.

Roux. Sur l'opération d'Alexander-Adam's. Revue médioale de la Suisse romande 1888. No. 11.

Sohroeder, C. Handbuch der Krankheiten der weiblichen Geschlechtsorgane. 1887.

Schultze, B. Zur Therapie hartnäckiger Retroflexion der Gebärmntter. Sammlung klin. Vorträge. Mai 1891. Centralbl. f. Gynäkologie 1879. No. 3.

$\mathrm{Schwartz}$, E. Du traitement des déplacements et des déviations utérines etc. Revue de chirurgie. 1889. p. 831.

Sänger, M. Ueber operativo Behandlung der Retroversio-flexio uteri. Centralblatt f. Gynäk. 1888. No. 2 und 3.

Sinclair, A. J. Bemerkungen über einen Fall von Alexander-Adamscher Operation (Edinb. med. Journ. 1885. Septbr. Ref. Centralbl. f. Gynäk. 1886. No. 23).

Sperling. Ventrofixatio uteri retroflexi. Deutsoh. med. Woehenschr. 1891. p. 179.

Strelin, A. Die ventrale Fixation als Mittel zur Hebung des Prolapsus uteri. Bern. J. D. 1890.

Tillaux, P. Traité d'anatomie topographique. 1884. pág, 894.

Veit, J. Die Indicationsstellung für die Behandlung der Retroflexio uteri (ref. Centralbl. f. Gynäk. No. 44. 1890).

Vuillet. Berl, klin. Wochenschr. 1891. No. 14.

Winckel. Lehrbuch der Franenkrankheiten. 1886. p. 363.

Wolf, K. Ueber ventrale Fixation bei Lageveränderungen des Uterus. J. D. Basel 1892.

$Z_{w}$ eifel, P. Lehrbuch der Geburtshülfe. 1887. pag. 304.

Zweifel, P. Ueber die Vaginalfixatio nteri, oder die Schücking'sche Vaginalligatur. Centralbl. f. Gynäk. 1890. No. 39. 